\title{
USE OF CONDUCTIMETRIC TECHNIQUE FOR DATA CAPTURE IN PREDICTIVE MICROBIOLOGY
}

\author{
E. SZIGETI ${ }^{\mathrm{a}, \mathrm{c}}$ and J. FARKAS ${ }^{\mathrm{b}}$ \\ ${ }^{a}$ Department of Microbiology and Biotechnology, Faculty of Food Sciences, Szent István University, \\ H-1118 Budapest, Somlói út 14-16. Hungary \\ ${ }^{\mathrm{b}}$ Department of Refrigeration and Livestock Products Technology, Faculty of Food Sciences, \\ Szent István University, H-1118 Budapest, Ménesi út 45. Hungary
}

(Received: 4 February 2000, accepted: 23 March 2000)

Conductimetry as an alternative data capture method for following microbial growth has a great potential as a research tool of predictive microbiology. In spite of this fact there is only a limited number of applications using conductimetric data for model generation. In this study the growth of single strains of Listeria monocytogenes and Lactococcus lactis was tested in 5 media using a RABIT ${ }^{\circledR}$ instrument. The goal of the work was to find selective growth media for Listeria and Lactococcus, respectively, in order to study their interaction in mixed-culture using the conductimetric technique. Whitley Anaerobic broth, Whitley Impedance broth and modified Whitely Impedance broth (Whitley Impedance broth + Chloramphenicol $7 \mathrm{mg} \mathrm{1}^{-1}$ ) were not suitable for following selectively the growth of Lactococcus lactis or Listeria monocytogenes in a mixed culture of the two bacteria. BiMedia $630 \mathrm{~A}$ for Lactococcus lactis and Bimedia $403 \mathrm{~A}$ for Listeria monocytogenes satisfied the demands raised by conductance measurement. Linear correlations were established between the graphically estimated TTD values of the conductance curves and the logarithmic numbers of colony forming units (CFU). The correlations were very strong in each case (determination coefficients $\left(\mathrm{R}^{2}\right)$ of the linear regression were higher than 0.98 at both medium-strain combinations). However, in BiMedia 630 Listeria monocytogenes was capable of slow growth, therefore, this medium would be feasible for studying microbial interactions if only low concentrations of Listeria (less than $10^{6} \mathrm{CFU} \mathrm{\textrm { } ^ { - 1 }}$ ) were present in the mixed culture.

Keywords: conductimetric measurement, RABIT, Lactococcus lactis, Listeria monocytogenes, selective media

For model generation in predictive microbiology large amounts of microbial growth data are required. Most mathematical models describing growth of microorganisms have been developed from viable cell-count data. Since the traditional

${ }^{\mathrm{c}}$ To whom the correspondence should be addressed. Szent István University,

Faculty of Food Sciences, Department of Microbiology and Biotechnology, Somlói út 14-16,

1118 Budapest, Hungary. Phone: +36-1-373-6201, Fax: +36-1-372-6340,

E-mail: e500eszi@omega.kee.hu 
viable count methods are labour- and material-intensive, and time-consuming, rapid instrumental methods seem to be attractive for capturing data used in model construction. Impedimetry as a versatile, less labourintensive and rapid method is promising for monitoring microbial growth. Impedimetric methods measure changes in electrical impedance, conductance or capacitance resulting from microbial growth in suitable medium, because during microbial metabolism large uncharged or weakly charged substrates (e.g. proteins, carbohydrates, lipids) are transformed into small highly charged end products (e.g. amino acids, lactate, acetate) which increase the conductivity of the growth medium.

Conductance measurements have been applied in a wide range of studies. For example, they were used to estimate microbial contamination of food (RULE, 1997) and to monitor selective activity of different groups of bacteria in a mixed-strains starter culture (SVENSSON, 1994). VIDÁCS and BECZNER $(1996,1998$ a) studied the effects of combined treatments on spore forming bacteria with the help of conductance measurements. DEÁK and BEUCHAT (1993a) evaluated the indirect conductance method for detection of yeasts. A selective medium for isolation Listeria monocytogenes from minced meat by an impedance method was developed by BAUMGART and co-workers (1994). VIDÁCS and BECZNER (1998b) compared the efficiency of different media for the enumeration of Clostridium sporogenes by direct conductance measurement. Since the conductimetric technique measures metabolic activity and integrate the effect of the number of organisms with their metabolic activity, it seems to be the most suitable tool for shelf-life prediction. BISHOP and co-workers (1984) applied an impedance test to predict the shelf-life of pasteurized milk. In spite of the fact that impedimetry can be used to provide a large number of data points in a relatively short time for developing predictive models, there is only a limited number of studies using impedimetrically measured data for model generation (BORCH \& WALlentin, 1993; DEÁK \& BEUCHAT, 1993 b-c, 1994; LINDBERG \& BORCH, 1994). The reason for this can be the lack of suitable selective media and methods to determine the growth characteristics of specific groups of microorganisms.

The aim of the present work was to test various media for selective estimation of Listeria monocytogenes and Lactococcus lactis, respectively, in order to study their interaction in a mixed-culture using conductimetric measurements.

\section{Materials and methods}

\subsection{Test organisms}

The following strains were tested: Lactococcus lactis SL05 (ARILAIT, France) Listeria monocytogenes NTCT pGC4/lux AB/(University of Nottingham, UK) 


\subsection{Impedimetric media}

The following media were tested for conductimetric measurements : Whitley Anaerobic broth (Don Whitley Scientific Ltd., UK), Whitley Impedance broth (Don Whitley Scientific Ltd., UK), Whitley Impedance broth + Chloramphenicol $\left(7 \mathrm{mg} \mathrm{l}^{-1}\right)$, BiMedia 630 A (Sy-Lab Vertriebs-Gesellschaft, Austria), and BiMedia 403 A (Sy-Lab Vertriebs-Gesellschaft, Austria). All media were sterilised at $121^{\circ} \mathrm{C}$ for $15 \mathrm{~min}$ before being used for conductance measurements.

\subsection{Inoculum}

The L. lactis inoculum was grown in MRS Bouillon (Merck, Germany), $L$. monocytogenes in BHI broth (Oxoid M225, UK) at $30^{\circ} \mathrm{C}$. In all experiments, $24 \mathrm{~h}$ cultures were used and the viable cell counts of samples inoculated into the impedimetric media were determined by the spread-plate technique using Brain Heart Infusion Agar (Oxoid M225, UK).

\subsection{Conductimetric measurements}

Direct conductrimetric measurements were performed with a RABIT ${ }^{\circledR}$ instrument (Don Whitley Scientific Ltd., West Yorkshire, UK), where 32 samples can be kept simultaneously in one incubator block.

Decimal dilutions of the $24 \mathrm{~h}$ cultures of test-organisms were prepared in peptone water and $0.5 \mathrm{ml}$ of each dilution was added to $4.5 \mathrm{ml}$ conductimetric medium investigated in the RABIT ${ }^{\circledR}$ tubes. All samples were analysed in triplicate at $30{ }^{\circ} \mathrm{C}$ incubation for $48 \mathrm{~h}$.

The change of conductance was measured automatically and registered every 6 min by the RABIT ${ }^{\circledR}$ computer system. Time to Detection (TTD) required to reach the detection limit for a change in conductance was determined.

\section{Results and discussion}

In our study the growth of Listeria monocytogenes and Lactococcus lactis in 5 media was monitored. In Whitley Anaerobic broth Listeria was not able to grow. Lactococcus lactis gave a conductance signal, however, it was weak and irregular. Taking this fact into account, further examination was not carried out with this medium. In the case of Whitley Impedance broth, both of the bacteria grew well, but TTD fluctuated erratically. Because we looked for selective media, further tests were not performed in this medium. 
Since the strain of Listeria monocytogenes used in this work was resistant to choramphenicol we decided to add this antibiotic to the Whitley Impedance broth, hereby making selective the medium for growth from mixed cultures with Lactococcus. As it was expected, chloramphenicol inhibited the growth of Lactococcus lactis whereas Listeria monocytogenes was capable of growth in this modified Whitley Impedance broth. However, the absolute changes in conductance proved to be too small for generating suitable TTD values.

The two selective media offered by Sy-Lab (VertriebsgmbH, Austria) for detection of either Listeria species or lactic acid bacteria seemed to be better for selective monitoring of growth of these microorganisms. Since these media were developed especially for the BacTrac impedimetric instrument which employs four electrodes (one pair for measuring changes in the medium, the other for electrode changes) it could not be excluded that they might behave differently if they are used with other instruments. The Lactococcus lactis strain tested grew well in BiMedia $630 \mathrm{~A}$ composed for detection lactic acid bacteria (LAB). Unfortunately, a relatively large temporary change was noted in conductance at the beginning of the measurement, which caused an early unrealistically short automatic TTD value. Thus, it was necessary to estimate reasonable TTD values by evaluation of the whole conductance curves. We determined the time periods needed for 100, 400 and $600 \mu \mathrm{S}$ conductance increases, respectively. Linear correlations were established between these corrected TTD values and the logarithm of colony forming units (CFU) determined by conventional spreadplate technique in the range of $2-8 \log _{10}$ CFU if we used the TTD values belonging to 400 and $600 \mu \mathrm{S}$ conductance increases. Figures 1 and 2 represent the relationships between the corrected TTD value read directly from the curves and initial $\log _{10}$ CFU per $\mathrm{ml}$. The TTD values belonging to $100 \mu \mathrm{S}$ conductance increase were not suitable for producing good correlation whereas determination coefficients $\left(\mathrm{R}^{2}\right)$ of the linear regressions were higher than 0.98 in the case of 400 and $600 \mu \mathrm{S}$ conductance increases. These results suggest that BiMedia $630 \mathrm{~A}$ seems to be a relatively good medium for monitoring the growth of Lactococcus lactis. However, taking into account the fact that Listeria also was capable of slow growth in this medium (approximately $10^{6}$ viable cell count were detected after $29 \mathrm{~h}$ ), for studies of microbial interactions BiMedia $630 \mathrm{~A}$ would be less suitable when high concentrations of Listeria would be present in the mixed culture.

Good results were achieved with the Listeria selective medium BiMedia $403 \mathrm{~A}$. Because this medium also underwent a relatively large temporary change in conductance at the beginning of the impedimetric measurement, it was also necessary to determine TTD values graphically. To achieve a strong correlation we used the graphically estimated TTD values belonging to 100,400 and $600 \mu \mathrm{S}$ conductance increases for calibration. 


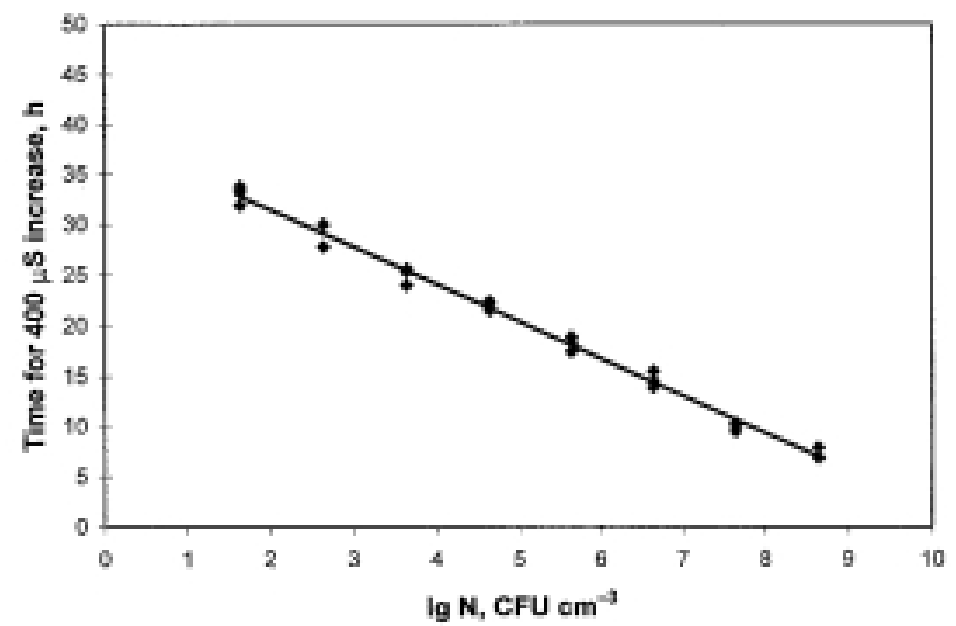

Fig. 1. Relationship between the time period needed for $400 \mu \mathrm{S}$ conductance increase and the $\log _{10} \mathrm{CFU} \mathrm{ml} l^{-1}$ in case of Lactococcus lactis at $30^{\circ} \mathrm{C}$ in BiMedia $630 \mathrm{~A}(\mathrm{n}=23) ; \mathrm{y}=-3.6812 \mathrm{x}+38.832 ; \mathrm{R}^{2}=0.991$

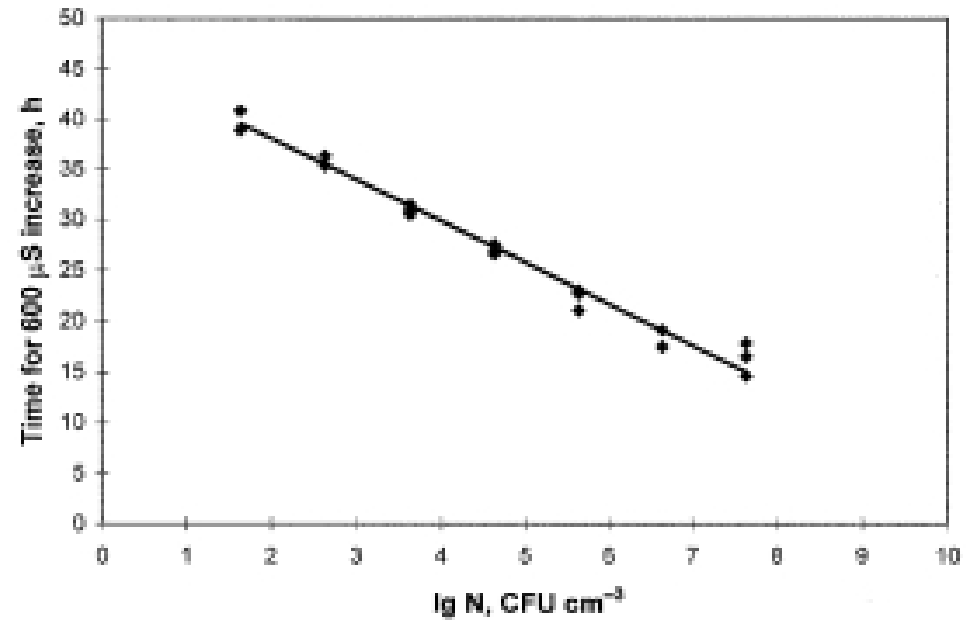

Fig. 2. Relationship between the time period needed for $600 \mu \mathrm{S}$ conductance increase and the $\log _{10} \mathrm{CFU} \mathrm{ml}{ }^{-1}$ in case of Lactococcus lactis at $30^{\circ} \mathrm{C}$ in BiMedia $630 \mathrm{~A}(\mathrm{n}=21) ; \mathrm{y}=-4.0929 \mathrm{x}+46.278 ; \mathrm{R}^{2}=0.9824$ 
In each case there was a linear relation between the corrected TTD value and the initial cell count. The regression lines are shown in Figs 3-5. Determination coefficients $\left(\mathrm{R}^{2}\right)$ of the linear regressions were higher than 0.98 in each case. In addition, Lactococcus was not able to grow in this medium. These results indicate that BiMedia $403 \mathrm{~A}$ seems to be a promising impedance medium for generating a large number of data points to describe the growth characteristic of Listeria in a mixed culture with Lactococcus lactis.

In the literature only a few media are reported to be suitable for selective monitoring of Listeria spp. using impedimetric measurements. PHILLIPS and GRIFFITHS (1989) evaluated 11 different selective formulations and demonstrated that a selective medium (AC broth) containing acriflavin, ceftazidine, nalidixic acid and aesculin gave good selectivity when testing pure cultures of Listeria ssp. LITMA broth for isolation Listeria monocytogenes from minced meat by impedance method was developed by BAUMGART and co-workers (1994). Only a low number of selective media is available for detection of lactic acid bacteria. Most of them are offered by the firms producing impedance analysers and are not developed especially for selective monitoring of LAB but generally for detection of anaerobic microorganisms. In addition, these media are so composed that they achieve a good result only using the same instrument which they were developed for. Our results with BiMedia $630 \mathrm{~A}$ seem to confirm these above mentioned facts.

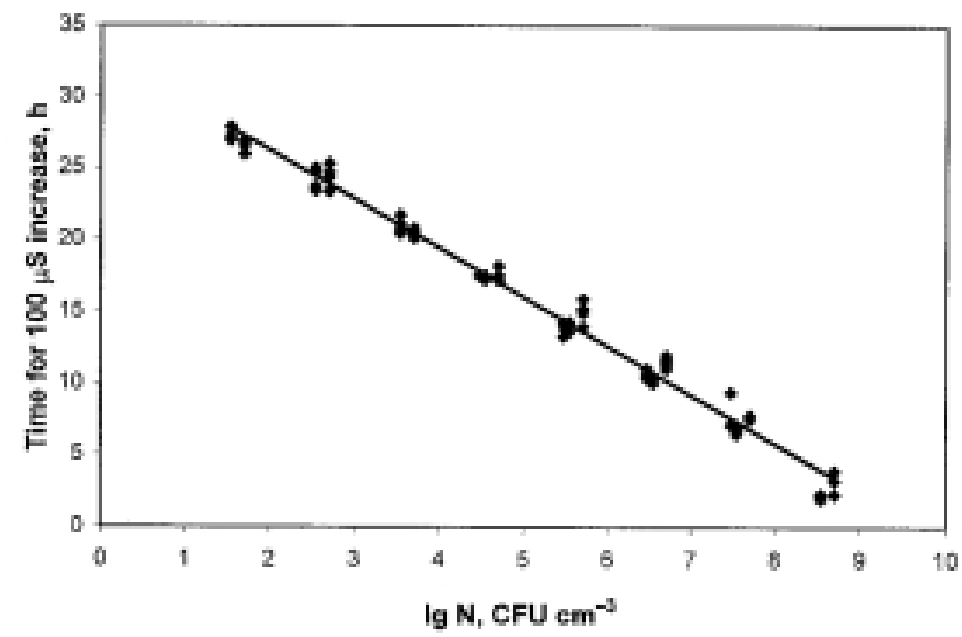

Fig. 3. Relationship between the time period needed for $100 \mu \mathrm{S}$ conductance increase and the $\log _{10} \mathrm{CFU} \mathrm{ml}{ }^{-1}$ in case of Listeria monocytogenes at $30{ }^{\circ} \mathrm{C}$ in BiMedia $403 \mathrm{~A}(\mathrm{n}=69) ; \mathrm{y}=-3.4093 \mathrm{x}+33.059 ; \mathrm{R}^{2}=0.9856$ 


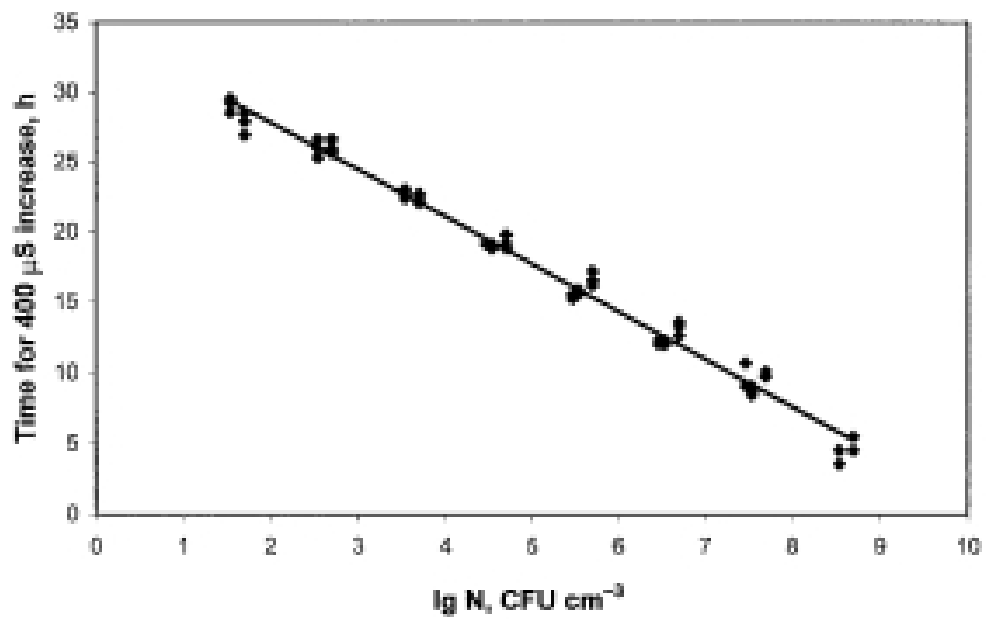

Fig. 4. Relationship between the time period needed for $400 \mu \mathrm{S}$ conductance increase and the $\log _{10} \mathrm{CFU} \mathrm{m}{ }^{-1}$ in case of Listeria monocytogenes at $30{ }^{\circ} \mathrm{C}$ in BiMedia $403 \mathrm{~A}(\mathrm{n}=69) ; \mathrm{y}=-3.3656 \mathrm{x}+34.551 ; \mathrm{R}^{2}=0.9872$

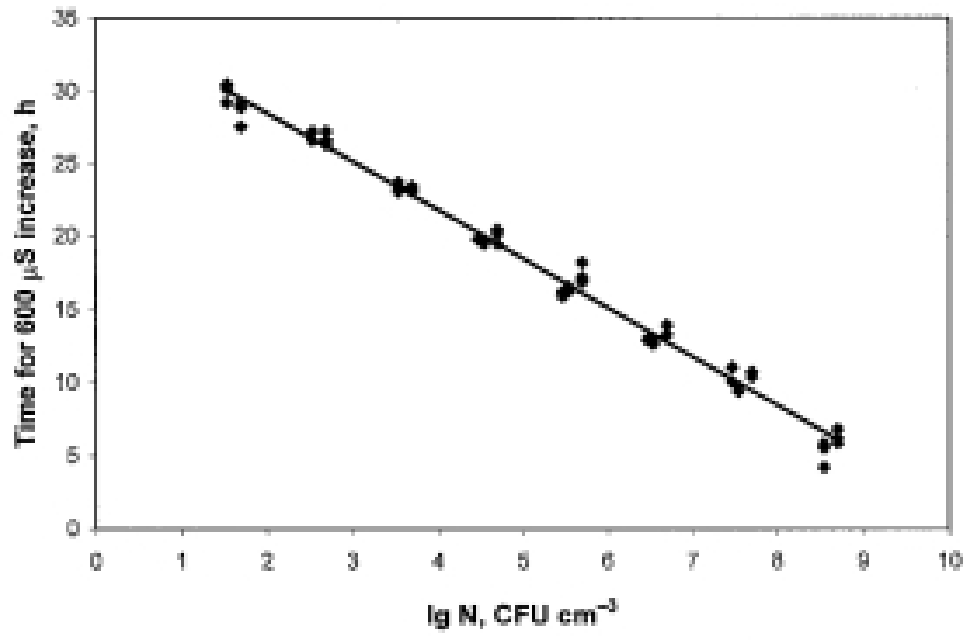

Fig. 5. Relationship between the time period needed for $600 \mu \mathrm{S}$ conductance increase and the $\log _{10} \mathrm{CFU} \mathrm{ml}{ }^{-1}$ in case of Listeria monocytogenes at $30{ }^{\circ} \mathrm{C}$ in BiMedia $403 \mathrm{~A}(\mathrm{n}=69) ; \mathrm{y}=-3.3425 \mathrm{x}+35.197 ; \mathrm{R}^{2}=0.9885$ 
These studies were a part of our contribution to the EU "PREMIUM" collaborative project (FAIR CT973129) and financially supported by a research contract of the National Commission for Technical Development (OMFB), Budapest and by the National Scientific Research Foundation (OTKA, grant No. T 024131).

\section{References}

BAUMGART, J., SIEKER, S. \& VOGELSANG, B. (1994): Listeria monocytogenes in Hackfleisch. Nachweis mit der Impedanz-Methode und einem neuen Selektivmedium. Fleischwirtschaft, 74, 647-648.

BISHOP, J. P., WHITE, C. R. \& FIRSTENBERG-EDEN, R. (1984): A rapid impedimetric method for determining the potential shelf-life of pasteurized whole milk. J. Fd Protection, 47, 471-475.

BORCH, E. \& WALLENTIN, C. (1993): Conductance measurements for data generation in predictive modelling. J. ind. Microbiol., 12, 286-290.

DEÁK, T. \& BEUCHAT, L. R. (1993a): Evaluation of the indirect conductance method for the detection of yeasts in laboratory media and apple juice. Fd. Microbiol., 10, 255-262.

DEÁK, T. \& BEUCHAT, L. R. (1993b): Comparison of conductimetric and traditional plating techniques for detecting yeasts in fruit juices. J. appl. Bacteriol., 75, 546-550.

DEÁK, T. \& BEUCHAT, L. R. (1993c): Use of indirect conductometry for predicting growth of food spoilage yeasts under various environmental conditions. $J$. ind. Microbiol., 12, 301-308.

DEÁK, T. \& BEUCHAT, L. R. (1994): Use of indirect conductometry to predict the growth of spoilage yeast, with special consideration of Zygosaccharomyces bailii. Int. J. Fd Microbiol., 23, 405-417.

LINDBERG, C. W. \& BORCH, E. (1994): Predicting the aerobic growth of Y. enterocolitica O3 at different pHvalues, temperatures and L-lactate concentrations using conductance measurements. Int. $J$. Fd Microbiol., 22, 141-153.

PHILLIPS, J. D. \& GRIFFITHS, M. W. (1989): An electrical method for detecting Listeria spp. Letters appl. Microbiol., 9, 129-132.

Rule, P. (1997): Measurement of microbial. activity by impedance. -in: TORTORELLO, M. L. \& GENDEL, S. M. (Eds) Food microbiological analysis. New technologies. Marcel Dekker, Inc., New York, pp. 305-314.

SVENSSON, U. K. (1994): Starter culture characterisation by conductance methods. J. Dairy Sci., 77, 3516-3523.

VIDÁCS, I. \& BECZNER, J. (1996): Effects of combined treatments on spore forming bacteria - Potentialities of the Malthus instrument. Acta Alimentaria, 25, 211-216.

VIDÁCS, I. \& BECZNER, J. (1998a): Application of conductance measurement for studying the combined effect of heat and $\mathrm{pH}$ on Clostridium sporogenes PA3679/S. De ware(n) Chemicus, 28, pp. 81-82.

VIDÁCS, I. \& BECZNER, J. (1998b): Comparison of media for enumeration of Clostridium sporogenes PA3679/S by conductance measurement. Acta Alimentaria, 27, 77-85. 